\title{
Incipient chordoma: a report of two cases of early-stage chordoma arising from benign notochordal cell tumors
}

\author{
Takehiko Yamaguchi $^{1}$, Hiroaki Watanabe-Ishiiwa ${ }^{2}$, Seiichiro Suzuki $^{3}$, Yoshihiko Igarashi ${ }^{4}$ \\ and Yoshihiko Ueda ${ }^{1}$ \\ ${ }^{1}$ Department of Pathology, Koshigaya Hospital, Dokkyo University School of Medicine, Minami-Koshigaya, \\ Koshigaya, Saitama, Japan; ${ }^{2}$ Department of Pathology, Dokkyo University School of Medicine, Mibu, Tochigi, \\ Japan; ${ }^{3}$ Department of Anatomic and Diagnostic Pathology, Dokkyo University School of Medicine, Mibu, \\ Tochigi, Japan and ${ }^{4}$ Dokkyo University School of Medicine, Mibu, Tochigi, Japan
}

\begin{abstract}
Chordomas are rare malignant bone tumors primarily involving both ends of the axial skeleton that present as destructive bone lesions with a large soft tissue mass. Chordomas were previously believed to arise from notochordal remnants. However, recent studies suggest the possibility that chordomas arise from benign notochordal cell tumors. We present two cases of coccygeal incipient chordoma that strengthen the new hypothesis. The first case was an 83-year-old man who died of prostatic adenocarcinoma. The second case was a 79-year-old man who died of hepatocellular carcinoma. The coccygeal tumors were composed of intraosseous and extraosseous infiltrative lesions. The intraosseous lesions consisted of both benign notochordal cell tumor and incipient chordoma. The extraosseous lesions were consistent with incipient chordoma. In addition, two other small benign notochordal cell tumors were found at a different level in case 1. It is conceivable that pre-existing intraosseous benign notochordal cell tumors transform into incipient chordoma and then extend through the cortex into the surrounding soft tissue. The incidence of incipient chordoma appears much higher than expected because chordomas are rare tumors with an incidence of one case per 1000000 persons per year. We suspect that unknown factors transform incipient chordoma into classic chordoma.
\end{abstract}

Modern Pathology (2005) 18, 1005-1010. doi:10.1038/modpathol.3800378; Published online 1 April 2005

Keywords: benign notochordal cell tumor; chordoma; incipient chordoma; malignant transformation; notochordal rest

Chordomas are rare malignant bone tumors primarily involving both ends of the axial skeleton. ${ }^{1-3}$ They usually present as destructive bone lesions with a large soft tissue mass. Chordomas are generally thought to arise from notochordal remnants. However, we documented a histologically confirmed case of classic chordoma arising in a precursor benign notochordal cell tumor and reported the clinicopathological details of intraosseous benign notochordal cell tumors. ${ }^{4-6}$ Based on the evidence, we concluded that intraosseous benign notochordal cell tumors are benign notochordal cell lesions with potential neoplastic characteristics and possibly

Correspondence: Dr T Yamaguchi, MD, PhD, Department of Clinical Pathology, Sapporo Medical University School of Medicine, S1W16, Chuo-ku, Sapporo 060-8543, Japan.

E-mail: takehiko@sapmed.ac.jp

Received 26 July 2004; revised and accepted 27 December 2004; published online 1 April 2005 serve as the real precursors of classic chordomas. We recently found two interesting cases of microscopic incipient chordomas coexistent with benign notochordal cell tumors in the coccyx, which supports the above hypothesis. This paper focuses on the histological details of incipient chordomas and provides additional possibility for oncogenetic mechanism of chordomas.

\section{Materials and methods}

\section{Case Report}

Case 1: An 83-year-old man died of prostatic adenocarcinoma. Autopsy examinations demonstrated metastatic tumors in the lungs and vertebral column.

Case 2: A 79-year-old man died of hepatocellular carcinoma with liver cirrhosis caused by hepatitis $\mathrm{C}$ virus infection. 


\section{Methods}

The vertebral column including sacrum and coccyx was sagittally dissected using an anterior approach to examine the metastatic spread of carcinoma to bone. Our institutional autopsy authorization permit specifically states that any part of the vertebra may be removed as a part of an unrestricted autopsy and examined microscopically. Each dissected specimen was cut into a 5-mm-thick slab with a Cutting Grinding System BS-3000 (EXAKT Apparatebau, Germany). The slabs were fixed with formalin and processed for routine microscopic examination following decalcification. Paraffin-embedded sections were immunohistochemically examined using the streptavidin-biotin-peroxidase method (Nichirei Co., Tokyo, Japan). The antibodies used are listed in Table 1. Microwave antigen retrieval was carried out prior to vimentin, AE1/AE3, CAM 5.2, and CK 18 immunostaining. To determine the specificity of immunostaining, we included known positive and negative tissues as controls.

\section{Results}

\section{Case 1}

The sagittally cut surface of the dissected vertebral column exhibited no apparent macroscopic abnormality except for osteoblastic metastatic tumors in the 12th thoracic vertebra and the second sacral vertebra. Careful microscopic examinations revealed five small notochordal cell lesions all within and around the coccyx (Figure 1). Two lesions were in the extraosseous soft tissue, and the other three were within the bone. The extraosseous lesions measured $20 \times 6 \mathrm{~mm}^{2}$ and $5 \times 1 \mathrm{~mm}^{2}$, respectively. They were not encapsulated. The larger lesion was located on the anterior aspect of the coccyx and extended around the distal end of the coccyx into the extraosseous adipose tissue and onto the peripheral nerve fibers (Figure 2a). The smaller lesion was located on the posterior aspect just beneath the ligament. They consisted of cords or strands of tumor cells set within a rich myxoid matrix (Figure $2 b)$. The tumor cells showed centrally or eccentrically located round nuclei and eosinophilic cytoplasm with occasional small vacuoles. The nuclei were slightly hyperchromatic and atypical. The intraosseous lesions measured up to $4 \times 2 \mathrm{~mm}^{2}$ in greatest dimension (Figure 1). The two smaller intraosseous lesions were composed of solid sheets of adipocyte-like vacuolated cells with eccentrically located round nuclei (Figure 3). The lesions lacked

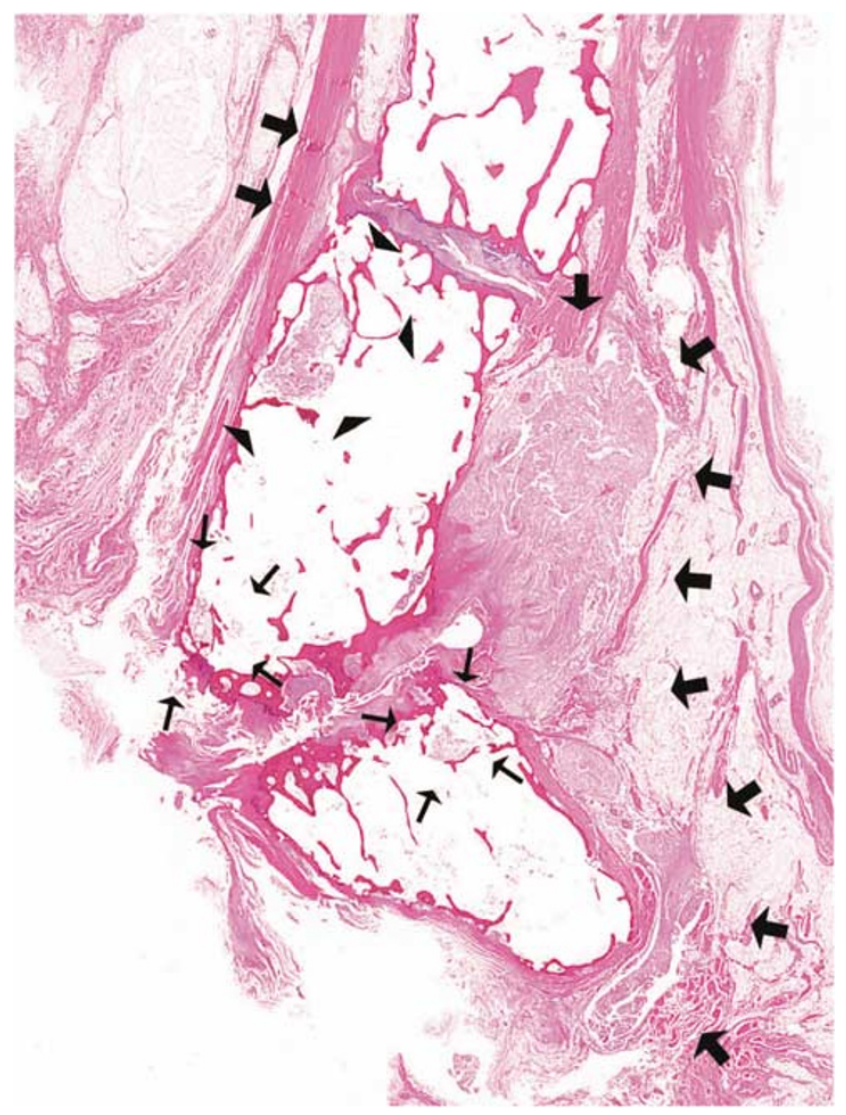

Figure 1 The coccyx obtained from an 83-year-old man who died of prostatic adenocarcinoma reveals three intraosseous and two extraosseous lesions (case 1). The extraosseous lesions are located on the anterior and posterior aspects of the coccyx and measure $20 \times 6 \mathrm{~mm}^{2}$ and $5 \times 1 \mathrm{~mm}^{2}$, respectively (thick arrows). The intraosseous lesions measure up to $4 \times 2 \mathrm{~mm}^{2}$ in greatest dimension. The largest intraosseous lesion is located in the posterior part of the coccyx, close to the smaller extraosseous lesion (arrow heads). The other lesions are located in the inferoposterior angle of the second distal coccygeal vertebra, where the largest lesion is located, and in the superoanterior angle of the most distal coccygeal vertebra (thin arrows).

Table 1 Antibodies used in the study

\begin{tabular}{|c|c|c|c|}
\hline Antibodies & Source & Clonality & Dilution \\
\hline Epithelial membrane antigen & DAKO Cytomation A/S, Glostrup, Denmark & $\mathrm{M}$ & $1: 100$ \\
\hline Cytokeratin (AE1/AE3) & DAKO Cytomation A/S, Glostrup, Denmark & M & $1: 100$ \\
\hline Cytokeratin (CAM5.2) & Becton Dickinson, San Jose, CA, USA & $\mathrm{M}$ & $1: 1$ \\
\hline Cytokeratin 18 & DAKO Cytomation A/S, Glostrup, Denmark & $\mathrm{M}$ & $1: 20$ \\
\hline Vimentin & DAKO Cytomation A/S, Glostrup, Denmark & $\mathrm{M}$ & $1: 100$ \\
\hline S-100 protein & Nichirei Co., Tokyo, Japan & $\mathrm{P}$ & $1: 200$ \\
\hline
\end{tabular}

M, monoclonal; P, polyclonal. 

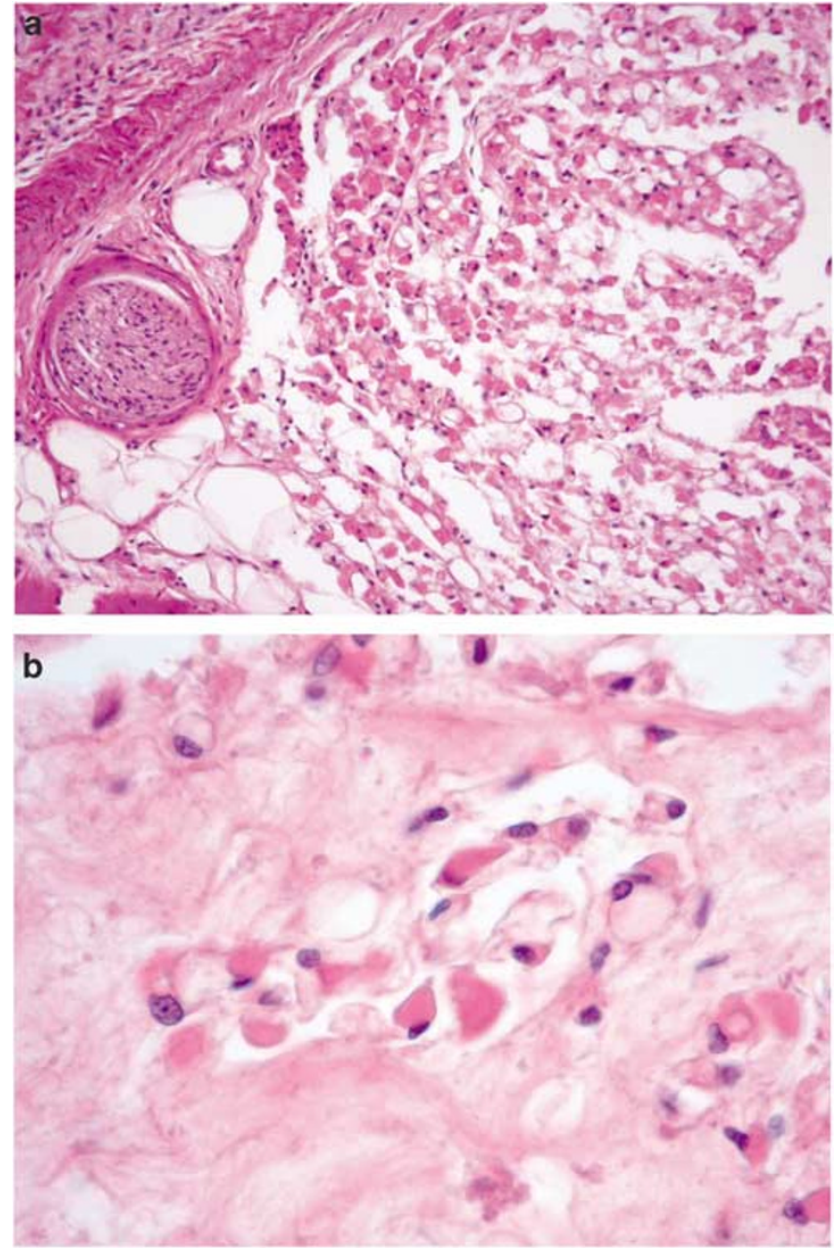

Figure 2 Soft tissue tumors around the coccyx. (a) The tumor lacks any fibrous capsule and extends into the extraosseous adipose tissue and close to the peripheral nerve fibers. These findings indicate the infiltrative potential of the tumor. (b) Highpower view reveals small nests or strands of tumor cells within a myxoid matrix. The tumor cells exhibit eccentrically located oval nuclei and eosinophilic cytoplasm. Some tumor cells contain large cytoplasmic vacuoles.

any intercellular myxoid matrix and significant nuclear atypia. These findings indicated benign notochordal cell tumors. The largest intraosseous lesion consisted of two parts with a relatively sharp interface (Figure 4). One was consistent with benign notochordal cell tumor, and the other was identical to the extraosseous lesions. The tumor cells of all lesions stained positive for EMA, AE1/AE3, CAM5.2, CK18, vimentin, and S-100 protein.

\section{Case 2}

The coccyx demonstrated an intraosseous notochordal lesion and an extraosseous lesion, measuring $3 \times 2 \mathrm{~mm}^{2}$ and $17 \times 8 \mathrm{~mm}^{2}$ in greatest dimension, respectively (Figure 5). The intraosseous lesion was located within the posterior part of the coccyx and

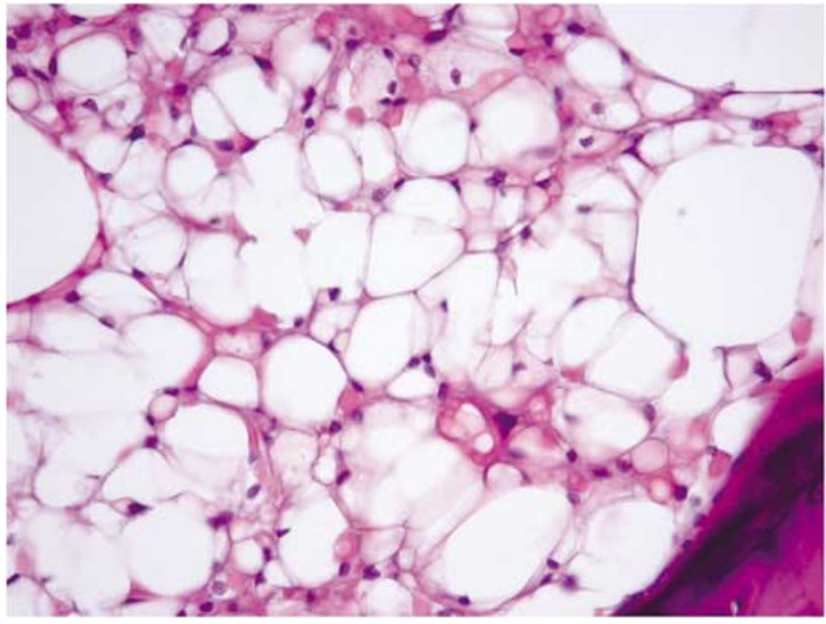

Figure 3 One of the smaller intraosseous tumors in the coccyx exhibiting a close pack of adipocyte-like vacuolated cells with eccentrically located bland nuclei. The lesion lacks any intercellular myxoid matrix or mitotic figures. The findings are typical for benign notochordal cell tumor.

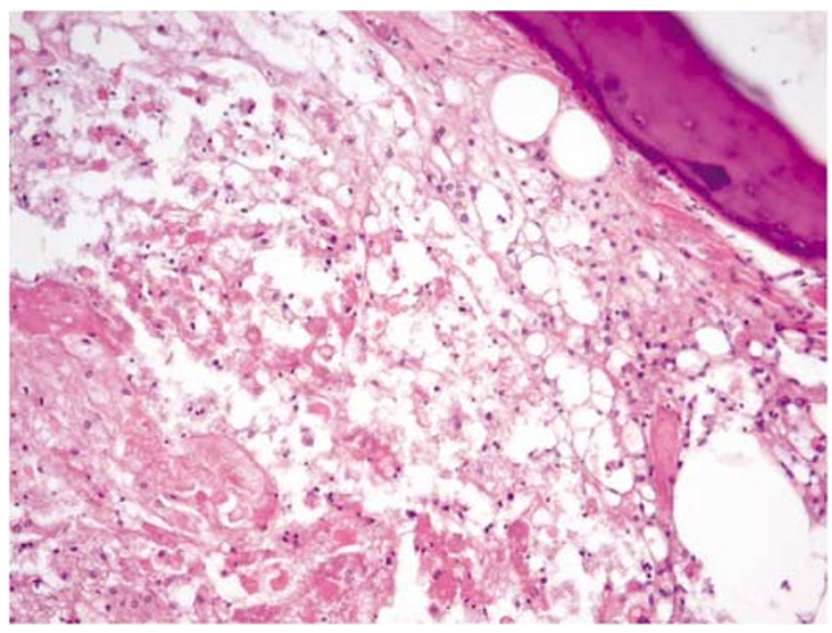

Figure 4 The largest intraosseous tumor in the coccyx consisting of two different components with a relatively sharp interface: a nonmyxoid tumor tissue along the bone trabecula and myxoid on the left. The nonmyxoid tumor demonstrates a close pack of vacuolated cells with bland round nuclei indicating benign notochordal cell tumor. The myxoid tumor reveals individual less vacuolated cells set within a rich myxoid matrix. The nuclei are slightly enlarged and hyperchromatic. The features are identical to those of the soft tissue lesions.

was composed of two components (Figure 6a). One component consisted of solid sheets of extremely vacuolated tumor cells with eccentrically located round nuclei and lacked any intercellular myxoid matrix (Figure 6b). The other demonstrated cords or strands of less vacuolated eosinophilic cells with slightly atypical round or oval nuclei embedded within a myxoid background. The extraosseous lesion, which was located on the posterior aspect of the coccyx just behind the intraosseous lesion, extended down through the subligament space and into the coccygeal intervertebral disk. The lesion 


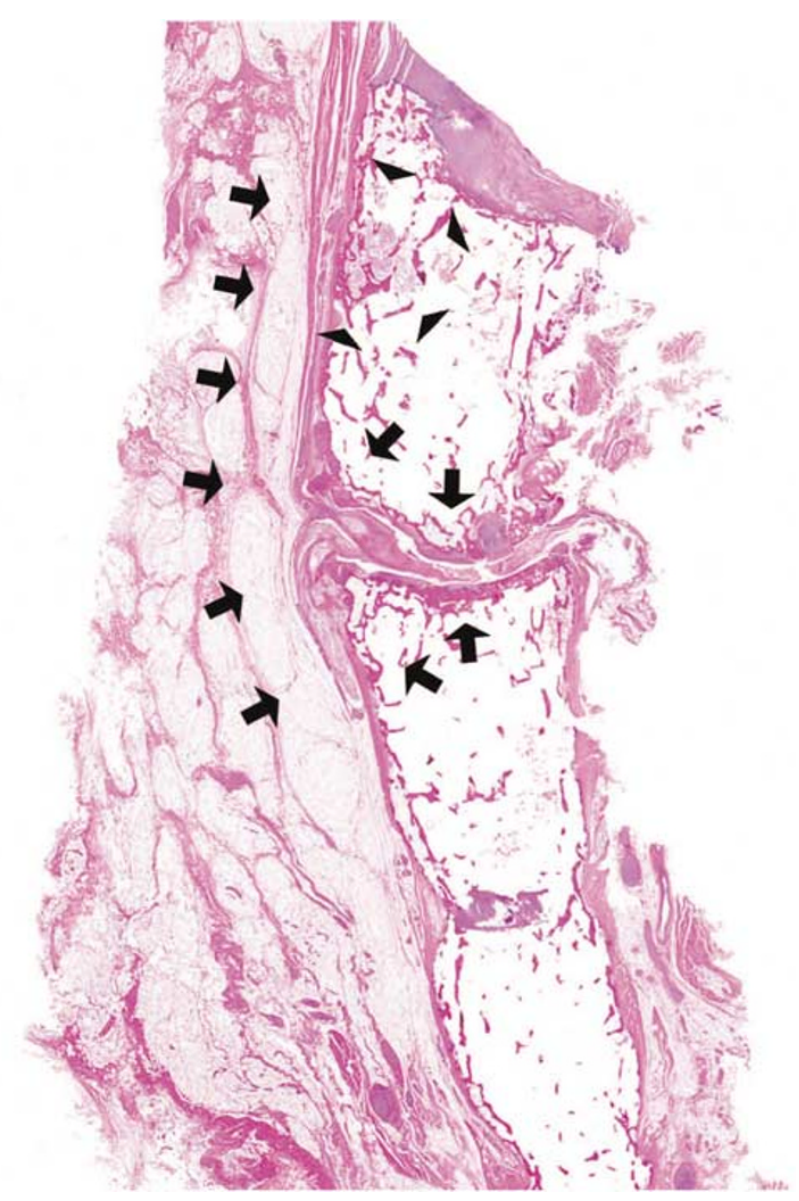

Figure 5 The coccyx dissected from a 79-year-old man who died of hepatocellular carcinoma with liver cirrhosis demonstrates an intraosseous lesion in the posterior part (arrow heads) and an infiltrative soft tissue lesion on the posterior aspect just behind the intraosseous lesion (thick arrows), measuring $3 \times 2 \mathrm{~mm}^{2}$ and $17 \times 8 \mathrm{~mm}^{2}$ in greatest dimension, respectively (case 2).

consisted of small nests or cords of eosinophilic tumor cells, some with small cytoplasmic vacuoles, within a myxoid matrix (Figure 6c). Some nests of the tumor cells were separated by thin fibrous septa and formed small lobules, reminiscent of classic chordoma (Figure 6d). The nuclei were mildly enlarged and exhibited small nucleoli. All tumor cells of both lesions stained with EMA, AE1/AE3, CAM5.2, CK18, vimentin, and S-100 protein.

\section{Discussion}

Benign notochordal cell tumors are recently discovered benign tumors of notochordal cell origin., They are found in approximately $20 \%$ of autopsy cases and their anatomical characteristics are identical to that of classic chordomas. Benign notochordal cell tumors consist of sheets of adipocyte-like vacuolated or less vacuolated eosinophilic tumor cells with eccentrically located round nuclei and lack any myxoid matrix. Mitotic figures are not usually found. The affected bone trabeculae are often sclerotic. The tumor cells stain for the epithelial markers, vimentin, and S-100 protein. Benign notochordal cell tumors are histologically different from notochordal vestiges in the fetal intervertebral disks or classic chordomas.

It is out hypothesis that benign notochordal cell tumors are precursors of chordomas. This new explanation for the origin of chordomas may help to resolve some of the contradictions of the classic theory that chordomas arise from notochordal vestiges directly. Although $\mathrm{Jaffe}^{7}$ predicted the presence of intraosseous precursors, none have been documented by modern histological examination. We documented a histologically confirmed case of chordoma arising from a benign notochordal cell tumor in 2002. ${ }^{4}$ This previous case suggested a small intraosseous benign notochordal cell tumor (described as a benign notochordal lesion in the original paper) located adjacent to the coccygeal chordoma. In addition, two other small benign notochordal cell tumors were evident in the sacrum at a different level. However, we could not totally eliminate the possibility that de novo chordoma developed coincidentally in the vicinity of a preexisting benign notochordal cell tumor.

The present cases exhibited both extraosseous infiltrative and intraosseous lesions. The intraosseous lesions consisted of two components: one component was consistent with benign notochordal cell tumor, and the other exhibited proliferation of chordoid cells with minimal nuclear atypia accompanied by an intercellular myxoid matrix. The interface between the two components appeared relatively sharp. The extraosseous tumors consisted of solid nests or cords of atypical chordoid cells set within a myxoid matrix variable in volume. In addition, two other small intraosseous benign notochordal cell tumors were evident in case 1 . These findings suggest that pre-existing intraosseous benign notochordal cell tumors transformed into an incipient chordoma that extended throughout the cortex into the subligament space, and infiltrated soft tissue or the intervertebral disk.

Benign notochordal cell tumors may be associated with small soft tissue components. ${ }^{6}$ They are located on the periosteum just above intraosseous lesions. Their histology is identical to that of intraosseous benign notochordal cell tumors. In contrast, the soft tissue components in the cases presented here were microscopically different from benign notochordal cell tumors. Furthermore, careful observation reveals that the small nests in the soft tissue components seemed to invade between or among the original anatomical structures. Therefore, the soft tissue components in the present cases are considered miniature chordomas.

Congdon ${ }^{8}$ documented four cases of notochordal cell lesions that were classified as benign chordoma. His first three cases are consistent with ecchordosis physaliphora sphenooccipitalis. The fourth case 

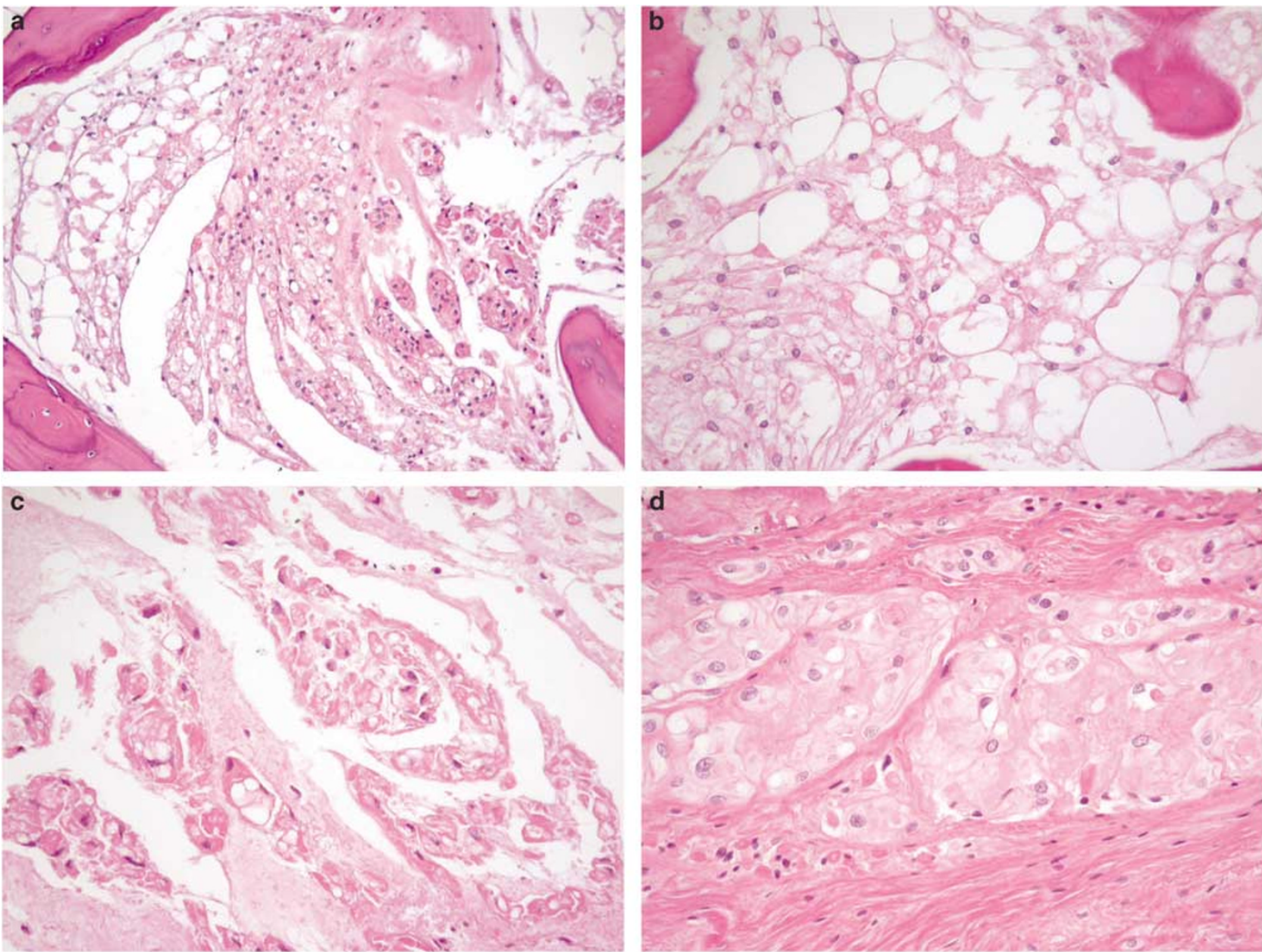

Figure 6 (a) The intraosseous lesion consists of a mixture of, on the left, benign notochordal cell tumor and, on the right, incipient chordoma with a relatively distinct margin. (b) High-power view of benign notochordal cell tumor demonstrates a close pack of vacuolated tumor cells with eccentrically located round nuclei. No intercellular myxoid matrix is recognized. (c) Close-up view of the extraosseous lesion exhibits strands of tumor cells or individual tumor cells embedded within a myxoid background. The tumor cells show slightly hyperchromatic nuclei and less vacuolated eosinophilic cytoplasm. (d) Small nests of the epithelioid tumor cells are separated by thin fibrous septa and form a lobular configuration, reminiscent of classic chordoma.

was a 44-year-old man who underwent coccygectomy for rectal carcinoid tumor. Serial sections of the coccyx demonstrated a notochordal lesion in the coccygeal intervertebral disk, which extended beneath the coccygeal periosteum. No information on coccygeal bone marrow was available. The description seems similar to the incipient chordoma presented in case 2. Benign notochordal cell tumors are usually located within bone and never infiltrate surrounding soft tissue even if they are associated with a small soft tissue mass on the periosteum. ${ }^{6}$ It is more likely that the lesion came from the periosteum into the intervertebral disk like the incipient chordoma in case 2 .

The incipient chordomas in our two cases were characterized by small infiltrative tumors composed of less vacuolated chordoid cells with an intercellular myxoid matrix. The nuclei were slightly larger and minimally atypical than those of benign notochordal cell tumors. The tumor cells were occasionally arranged in a small lobular configuration. However, the tumors did not show any large lobular configuration or the wide variety of nuclear atypia that are typical of classic chordomas. The incipient chordomas presented here strengthen the new theory that chordomas arise from pre-existing intraosseous benign notochordal cell tumors. The term of 'incipient chordoma' includes two meanings. One indicates miniature chordomas and the other may mean prechordomatous intermediate conditions between benign notochordal cell tumor and classic chordoma. The incidence of incipient chordoma seems much higher than expected since these tumors were found over a period of approximately 3 months. In contrast, chordomas are extremely rare malignant bone tumors found in one case per 1000000 persons per year., ${ }^{9,10}$ The phenomenon of benign notochordal cell tumors 
undergoing transformation into incipient chordomas may be a relatively common event, and there may be some unknown factors necessary to transform incipient chordomas into classic chordomas. However, the confirmation of this hypothesis will require further careful histological examination of a larger number of incipient chordomas.

\section{References}

1 Dorfman HD, Czerniak B. Bone Tumors. CV Mosby: St Louis, 1998, pp 974-1008.

2 Mirra JM, Rocca CD, Nelson SD, et al. Chordoma. In: Fletcher CDM, Unni KK, Martens F (eds). World Health Organization Classification of Tumours. Pathology and Genetics of Tumours of Soft Tissue and Bone. IARC Press: Lyon, 2002, pp 315-317.

3 Unni KK. Dahlin's Bone Tumors: General Aspects and Data on 11087 Cases, 5th edn. Lippincott-Raven: Philadelphia, 1996, pp 291-305.

4 Yamaguchi T, Yamato M, Saotome K. First histologically confirmed case of a classic chordoma arising in a precursor benign notochordal lesion: differential diagnosis of benign and malignant notochordal lesions. Skeletal Radiol 2002;31:413-418.

5 Yamaguchi T, Suzuki S, Ishiiwa H, et al. Intraosseous benign notochordal cell tumours: overlooked precursors of classic chordomas? Histopathology 2004;44: 597-602.

6 Yamaguchi T, Suzuki S, Ishiiwa $\mathrm{H}$, et al. Benign notochordal cell tumors: a comparative histological study of benign notochordal cell tumors, classic chordomas and notochordal vestiges of fetal intervertebral discs. Am J Surg Pathol 2004;28:756-761.

7 Jaffe HL. Tumors and Tumorous Conditions of the Bones and Joints. Lea \& Febiger: Philadelphia, 1958, pp 451-461.

8 Congdon CC. Benign and malignant chordomas: a clinico-anatomical study of twenty-two cases. Am J Pathol 1953;28:793-821.

9 Dorfman HD, Czerniak B. Bone cancers. Cancer 1995;75:203-210.

10 McMaster ML, Goldstein AM, Bromley CM, et al. Chordoma: incidence and survival patterns in the United States, 1973-1995. Cancer Causes Control 2001;12:1-11. 